\title{
Altered Immunohistochemical Expression Patterns of HLA Class I during the Clinical Course of Cervical Intraepithelial Neoplasia
}

\author{
Okuto Koguchi ${ }^{1}$, Haruna Nishimaki ${ }^{2}$, Yoko Nakanishi², Hiroko Kobayashi ${ }^{2}$, \\ Sumie Ohni ${ }^{2}$, Xiaoyan Tang ${ }^{2}$, Yoshiaki Kusumi ${ }^{2}$ and Shinobu Masuda ${ }^{2}$ \\ ${ }^{1} 6$ th grade student, Nihon University School of Medicine, Tokyo 173-8610, Japan and ${ }^{2}$ Division of Oncologic Pathology, \\ Department of Pathology and Microbiology, Nihon University School of Medicine, Tokyo 173-8610, Japan
}

Received January 11, 2021; accepted February 26, 2021; published online April 17, 2021

\begin{abstract}
It is unclear how immunohistochemical expression patterns of HLA class I in the premalignant phase of cervical intraepithelial neoplasia (CIN) alter during the clinical follow-up period. The present study aimed to demonstrate the correlation between the immunohistochemical expression pattern of HLA class I and the CIN grade through repeated examinations during the clinical course. Expression patterns of HLA class I, p16INK4a, and PD-L1 were immunohistochemically examined using formalin-fixed paraffin-embedded (FFPE) sections of biopsy or conization samples that were obtained from 20 patients diagnosed with CIN. The mRNA expression levels of HLA class I were analyzed by real-time reverse transcription polymerase chain reaction using FFPE sections of 14 patients, who were examined metachronously during the follow-up period. HLA class I expression was limited to the lower part of the epithelial thickness (M1 pattern) in more than half of CIN1 cases, and was present throughout the epithelial thickness (M2 pattern) in one fourth of CIN1 and CIN2 cases approximately. Heterogeneous expression ( $\mathrm{H}$ pattern) was detected in half of CIN2 and CIN3 cases and in the all of squamous cell carcinoma cases. Metachronous examinations revealed that these immunohistochemical patterns altered more frequently than the CIN grade. The rate of change of HLA class I mRNA expression level was higher in cases with a progressed immunohistochemical pattern compared to those with regressed immunohistochemical pattern. In conclusion, the immunohistochemical pattern of HLA class I expression is associated with the CIN grade, and it is alterable during the clinical course, especially in CIN2.
\end{abstract}

Key words: human papilloma virus, cervical intraepithelial neoplasia, HLA class I, immunohistochemistry

\section{Introduction}

Approximately, 10,500 women are affected by uterine cervical cancer, and it is responsible for 2,900 deaths per year [1]. The most affected age was the early forties for uterine cervical cancer, which in contrast to the late fifties

Correspondence to: Shinobu Masuda, MD, PhD, Division of Oncologic Pathology, Department of Pathology and Microbiology, Nihon University School of Medicine, Oyaguchi Kami-cho, Itabashi-ku, Tokyo 173-8610, Japan. E-mail: masuda.shinobu@nihon-u.ac.jp for uterine body cancer [1]. The five-year relative survival rate was $95.3 \%$ for stage I, $78.7 \%$ for stage II, $61.4 \%$ for stage III, and $25.2 \%$ for stage IV cancer [1]. The etiology and development of uterine cervical cancer are closely related to human papilloma virus (HPV) infection. HPV alpha 5 (types 51), alpha 6 (type 56), alpha 7 (type 18, $39,45,59$ ), and alpha 9 (type 16, 31, 33, 35, 52, 58) are categorized as carcinogenic (International Agency for Research on Cancer category 1), and HPV 16 and 18 have the strongest carcinogenicity [14]. High-risk types of HPV infection induce persistent expression of HPV genes E6 and 
E7, which suppress the function of $\mathrm{p} 53, \mathrm{p} 21$, and $\mathrm{pRb}$ proteins and stimulate the development of cervical cancer [14].

HLA class I glycoproteins (HLA-A, -B, -C), encoded by major histocompatibility complex (MHC) on chromosome 6p21.3 and expressed on widely-distributed nucleated cells, play a major role in the immune system. Viruses attached onto the cell surface are internalized and destroyed, and their antigens are expressed on the HLA class I glycoproteins, which are dimerized with $\beta 2$ microglobulin at the cell membrane. Virus-infected cells activate immune reactions and produce cytokines such as interferon- $\gamma(\mathrm{IFN}-\gamma)$ and tumor necrosis factor- $\alpha$ (TNF- $\alpha$ ), leading to the upregulation of HLA class I in order to make infected cells susceptible to death by cytotoxic $\mathrm{T}$ cells (CTLs) [17]. However, multiple studies have suggested that HPV evades adaptive immune responses through several mechanisms [13]. After epithelial cells in the basal layer of the squamocolumnar junction are infected, HPV persists in epithelial cells with a low copy number of the HPV gene without disrupting the epithelial cells, and escapes recognition by the immune cells. HPV is carried upward to the surface of the epithelium by the differentiation of keratinocytes, after which is spreads to other epithelial cells. Another mechanism is that the E6 and E7 proteins, combined with interferon (IFN) regulatory factors (IRFs) downstream of the pathogen-associated molecular patterns (PAMPs) and pattern recognition receptors (PRRs) axis, suppresses the innate immune response by downregulating IFN gene transcription. Furthermore, HPV E7 protein reduces the expression of HLA class I and transporter associated with antigen processing (TAP) at the transcriptional level $[8,16]$. While the majority of previous studies on cervical cancer showed that HPV genes repress the expression of HLA class I, the effects of HPV on oncogenesis and immune evasion in pre-malignant lesions remains unknown.

In contrast to HLA class I, HLA class II (HLA-DP, $-D Q,-D R$, and -DN/-DO) are expressed in only a few cells, namely B lymphocytes, antigen-presenting cells (dendritic cells and Langerhans cells, monocytes, and macrophages), activated $\mathrm{T}$ cells, and some epithelial cells [7], and are involved in antigen presentation for T cell activation. HLA class II is not expressed in normal cervical squamous epithelium [9], but is upregulated in squamous cell carcinoma (SCC) of the uterine cervix. Immunohistochemical analysis showed that $52 \%$ of SCCs were positive for HLA class II [3]. The relationship between HLA polymorphisms for HLA class II and cervical SCC has been controversial, probably due to the ethnic diversities reported between countries, but DQB1, DRB1, and DR-DQ haplotypes (DRB1*1501-DQB1*0602) were most frequently reported to be correlated with cervical carcinoma [4]. Recent metaanalyses showed that HLA-DPB1 $(* 0301, * 0402, * 1301$, rs9277535, rs3117072) and HLA-DRB1 $(* 0301, * 0403$, $* 0404, * 0803, * 1312, * 1502$ ) were correlated with cancer risk $[2,19]$. Thus, the correlation between HLA class II system and cervical SCC has been investigated; however, it has not been clarified in the early phase of cervical intraepithelial neoplasia (CIN).

The present study aimed to examine how immune systems react to HPV infection in the pre-malignant phase of carcinogenesis. Therefore, we investigated the correlation between HLA class I expression and the CIN grade examined repeatedly during the follow-up period.

\section{Materials and Methods}

\section{Patient characteristics and processing of samples}

Among the patients who were examined by biopsy or underwent conization between 2009 and 2010 at Nihon University Hospital and who were diagnosed with uterine cervical intraepithelial neoplasia (CIN), twenty patients who were confirmed to be infected by HPV type 16, with or without additional types of HPV, were selected. The distribution of CIN diagnosed at first examination (CIN1 $(\mathrm{n}=7)$, CIN2 $(\mathrm{n}=8)$, and CIN3 $(\mathrm{n}=5))$, which was representative of the most progressed tumor grade among several obtained specimens, and the HPV typing are shown in Supplementary Table S1. Fourteen out of the 20 patients were repeatedly examined during the follow-up period by biopsy $(n=4)$ or conization $(n=10)$ at the second examination. Three non-neoplastic squamous epithelium samples and 4 SCC samples were examined as controls. If formalinfixed paraffin embedded (FFPE) sections, of which specimen was the representative most progressed CIN, were not large enough for evaluation by reverse transcription polymerase chain reaction (RT-PCR), a larger specimen among several specimens at the same examination were selected for extraction of mRNA. In total, 47 lesions were evaluated for immunohistochemical patterns and 28 lesions were evaluated for mRNA expression levels.

This study was conducted in accordance with the Declaration of Helsinki and the study design was approved by the institutional review board of the Nihon University Itabashi Hospital Ethics Committee (Approval numbers RK-181001-1).

\section{Immunohistochemistry (IHC) for HLA class I, p16, and PD- $L 1$}

IHC was performed on 4- $\mu \mathrm{m}$ FFPE tissue sections. After the tissue sections were dewaxed, antigen retrieval was performed.

Tissue sections were autoclaved in citric buffer $(\mathrm{pH}$ $6.0,105^{\circ} \mathrm{C}$ for $10 \mathrm{~min}$ ) for HLA class $\mathrm{I}$ and in Target Retrieval Solution buffer ( $\mathrm{pH}$ 6.1, Agilent Technologies, Inc., CA, USA) at $105^{\circ} \mathrm{C}$ for $10 \mathrm{~min}$ for PD-L1, and tissue sections were boiled in EDTA buffer $(\mathrm{pH} 9.0$, $97^{\circ} \mathrm{C}$ for $20 \mathrm{~min}$ ) for $\mathrm{p} 16 \mathrm{INK} 4 \mathrm{a}$. The sections were then cooled at room temperature $\left(20 \pm 3^{\circ} \mathrm{C}\right)$. After washing with phosphate-buffered saline (PBS, $\mathrm{pH} 7.2$ ), the sections were processed to block endogenous peroxidase activity with $0.3 \%$ hydrogen peroxide. The sections were then 


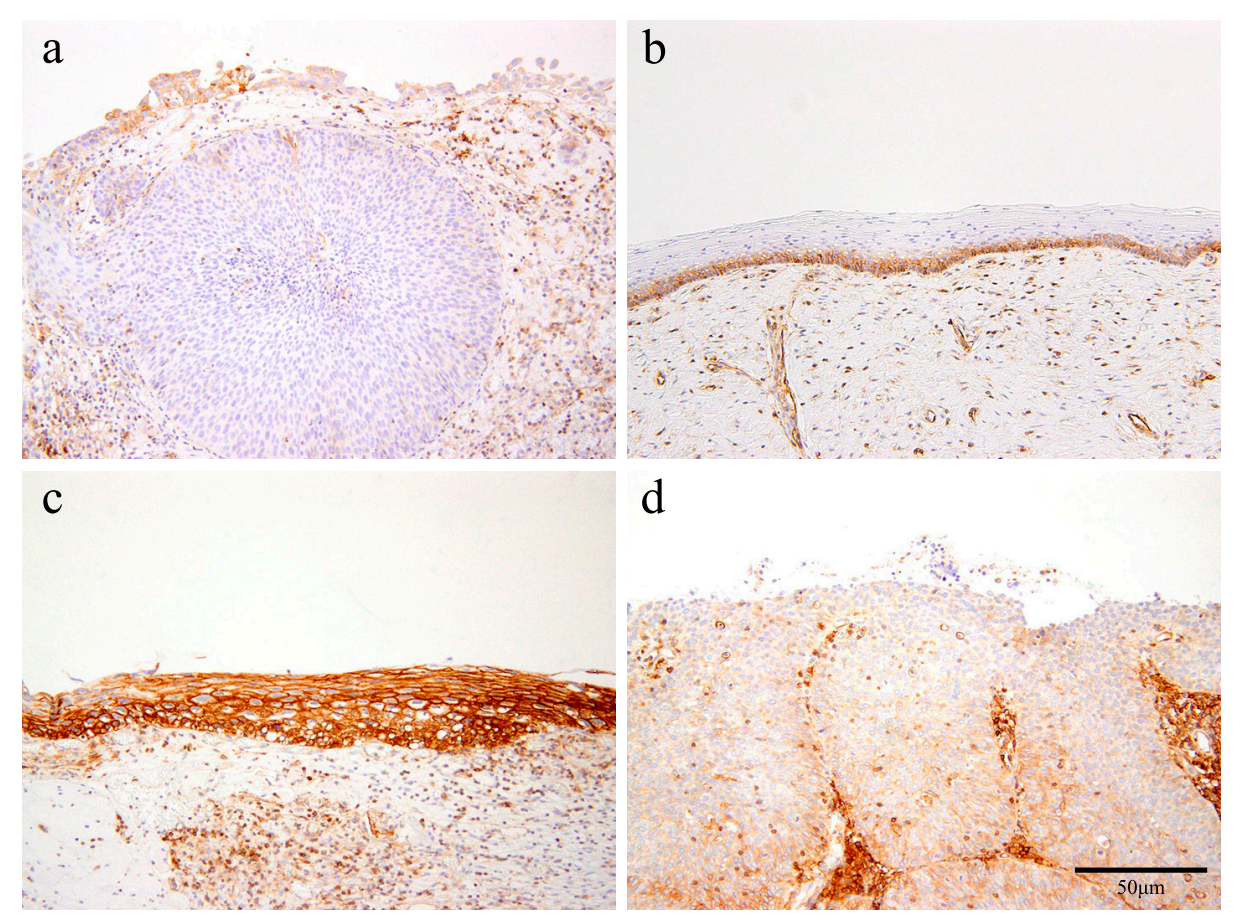

Fig. 1. Immunohistochemical expression pattern for HLA class I. Immunohistochemical staining of the cervical epithelium: (a) negative (N pattern), (b) positive at lower two-thirds of epithelial layer (M1 pattern), (c) positive for more than two-thirds of epithelial layer (M2 pattern), and (d) positive heterogeneously (H pattern), according to the criteria reported previously [8]. Bar $=50 \mu \mathrm{m}$.

incubated with the primary antibody for $30 \mathrm{~min}$ at RT. The primary antibodies used were anti-mouse monoclonal HLA Class I antibody (clone EMR8-5, ab70328, ×1/1000, Abcam plc., Cambridge, UK), which reacts with the heavy chains of human HLA class 1 A, -B, and -C; anti-mouse monoclonal p16INK4a antibody (clone E6H4, 705-4713, ready to use, F. Hoffmann-La Roche Ltd., Basel, Switzerland); and anti-rabbit monoclonal PD-L1 antibody (clone 28-8, $\times 1 / 400$, Abcam) (Supplementary Table S2). After washing with PBS, PD-L1 staining sections were incubated with Envision FLEX+ Rabbit LINKER (Ready to use, Agilent Technologies) for $15 \mathrm{~min}$ at RT. After washing with PBS, PD-L1 staining sections were incubated with Envision FLEX+ polymer (Agilent Technologies) for 30 min at RT. Both HLA class I and p16INK4a staining sections were incubated with Simple Stain MAX-PO (MULTI) (Nichirei Biosciences Inc., Tokyo, Japan) for $30 \mathrm{~min}$ at RT. They were washed with PBS and visualized using 3,3'diaminobenzidine tetrahydrochloride (DAB), and counterstained with hematoxylin. Clinical materials that were already known and confirmed to be positive were used as a positive control, and tissue sections were incubated with PBS and treated as a negative control for the following process.

Two certified pathologists (HN and SM) evaluated HLA class I expression pattern independently without clinical data or history. HLA class I expression patterns were classified into four categories based on the modified criteria by Cromme et al. as follows [3] — N: negative (completely negative to positive cells are less than 10\%), M1: when lower two-thirds of epithelial layer were stained positively and monotonously, M2: when more than two-thirds of epithelial layer were stained positively and monotonously, H: heterogeneously positive (Fig. 1).

p16INK4a expression was estimated as a 4-point score based on the fraction of positive cells in the epithelial layer where 0: negative, 1: positive cells are limited in less than one-third of the basal layer of epithelium, 2: positive cells are detected in less than two-thirds of epithelium, 3 : positive cells are detected in more than two-thirds of the epithelium. PD-L1 expression level was evaluated using tumor proportion score (TPS) - 0 , less than $1 \%$; 1 , less than $50 \%$; 2 , more than $50 \%$.

\section{Quantitative RT-PCR (qRT-PCR)}

FFPE tissue blocks were cut into $8 \mu \mathrm{m}$ sections and mounted on membrane film-coated glass slides; three serial sections were prepared. The FFPE sections were deparaffinized, hydrated, and stained with toluidine blue. After air-drying at RT, the cervical lesions (CIN/SCC) were separately microdissected using a laser microdissection instrument (PALM-MBIII-N, Carl Zeiss Microscopy, Munich, Germany).

Total RNA was extracted from the microdissected samples and cDNA was synthesized as previously described [17]. Initially, target sequences were amplified using the following primer sets for the RTPCR: HLA class I: forward, 5'-ccgaaattctccettgtatgtg-3', 
Table 1. Correlation between degree of cervical lesions (CIN/SCC) and immunohistochemical expression pattern of HLA class I

\begin{tabular}{|c|c|c|c|c|c|}
\hline \multirow{3}{*}{ IHC pattern } & \multicolumn{5}{|c|}{ No. of lesions } \\
\hline & Normal & CIN1 & CIN2 & CIN3 & $\mathrm{SCC}$ \\
\hline & $\mathrm{n}=3(\%)$ & $\mathrm{n}=13(\%)$ & $\mathrm{n}=18(\%)$ & $\mathrm{n}=9(\%)$ & $\mathrm{n}=4(\%)$ \\
\hline $\mathrm{N}$ & $1(33.3)$ & 0 & $1(5.6)$ & $3(33.3)^{\mathrm{a}}$ & 0 \\
\hline M1 & $2(66.7)$ & $9(69.2)^{\mathrm{a}}$ & $2(11.1)$ & 0 & 0 \\
\hline M2 & 0 & $3(23.1)$ & $5(27.8)$ & $1(11.1)$ & 0 \\
\hline $\mathrm{H}$ & 0 & $1(7.7)$ & $10(55.6)$ & $5(55.6)$ & $4(100)^{\mathrm{a}}$ \\
\hline
\end{tabular}

CIN, cervical intraepithelial neoplasia; SCC, squamous cell carcinoma; HLA, human leukocyte antigen. N, negative: M1, when lower two-thirds of epithelial layer were stained positively and monotonously: M2, when more than two-thirds of epithelial layer were stained positively and monotonously:

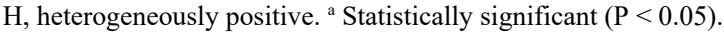

reverse 5'-tgcgatattcttccctcttcag-3', $\beta$-actin as internal control; forward 5-ggtcaccagggctgctttta-3, reverse 5ctcgetcctggaagatggtg- $3^{\prime}$. The thermal cycling profile was as follows: initial denaturation at $95^{\circ} \mathrm{C}$ for $10 \mathrm{~min}, 30$ cycles of denaturation at $94^{\circ} \mathrm{C}$ for $30 \mathrm{sec}$, annealing at $60^{\circ} \mathrm{C}$ for 30 sec, and extension at $72^{\circ} \mathrm{C}$ for $1 \mathrm{~min}$.

qRT-PCR was performed using $2 \mu \mathrm{L}$ of the first RTPCR products, TaqMan ${ }^{\circledR}$ probes (Thermo Fisher Scientific Inc., Waltman, MA, USA), TaqMan ${ }^{\circledR}$ gene expression Master Mix (Thermo Fisher Scientific Inc.), and the QuantStudio 3 real-time PCR system (Thermo Fisher Scientific Inc.). The premixed primers and probes used were as follows: HLA class I (Assay ID: Hs01058806_g1) and $\beta$-actin (Assay ID: Hs01060665_g1). The qRT-PCR thermal cycling profile was as follows: initial denaturation at $95^{\circ} \mathrm{C}$ for $10 \mathrm{~min}, 50$ cycles of denaturation at $95^{\circ} \mathrm{C}$ for $15 \mathrm{sec}$ and extension at $60^{\circ} \mathrm{C}$ for $1 \mathrm{~min}$. Each experiment was performed in duplicate. The relative expression level of HLA class I mRNA was normalized to the $\beta$-actin expression level by the $\Delta \mathrm{Ct}$ method.

\section{Statistical analysis}

Correlation between immunohistochemical expression pattern or score and the degree of cervical lesions was analyzed using Fisher's exact test. Correlation between HLA class I mRNA expression levels and the degree of cervical lesions was analyzed using the Kruskal-Wallis test.

\section{HPV genotyping}

HPV genotyping was performed by polymerase chain reaction-sequence specific oligonucleotide (PCRrSSO) methods using GenosearchTM HPV31 (Medical \& Biological Laboratories Co., Ltd, Nagoya, Japan) and Luminex $^{\circledR} 100 / 200 T M$ (Luminex, TX, USA) [20].

\section{Results}

\section{Correlation between immunohistochemical findings and CIN grade}

Immunohistochemical expression patterns of HLA class I in normal epithelium, CIN, and SCC samples are summarized in Table 1. Normal squamous epithelium showed a negative $(\mathrm{N})$ pattern $(1 / 3 ; 33.3 \%)$ or $\mathrm{M} 1$ pattern $(2 / 3 ; 66.7 \%)$, and no M2 or H pattern was detected. Majority of CIN1 showed M1 pattern $(9 / 13 ; 69.2 \%)$ with less M2 or $\mathrm{H}$ pattern $(\mathrm{P}<0.05)$. CIN2 showed an M2 pattern $(5 / 18 ; 27.4 \%)$ and an increased proportion of $\mathrm{H}$ pattern $(10 / 18 ; 55.6 \%)$. CIN3 showed an increased $\mathrm{H}$ pattern $(5 / 9$; $55.6 \%)$ and $\mathrm{N}$ pattern $(3 / 9 ; 33.3 \%)(\mathrm{P}<0.05)$. All the 4 SCC samples showed $\mathrm{H}$ patterns $(\mathrm{P}<0.05)$.

The distribution of $\mathrm{p} 16 \mathrm{INK} 4 \mathrm{a}$ expression in normal epithelium, CIN, and SCC samples are shown in Supplementary Table S3. All normal squamous epithelia were negative for $\mathrm{p} 16 \mathrm{INK} 4 \mathrm{a}$ expression $(\mathrm{P}<0.05)$. Eight out of $13(61.5 \%)$ CIN1 lesions were negative $(\mathrm{P}<0.05)$, and $4 / 13(30.8 \%)$ were estimated as score 2 , as defined in the Materials and Methods section. Thirteen out of $18(72.2 \%)$ CIN2 lesions were estimated as score $2(\mathrm{P}<0.05)$. CIN3 showed a score of 3 in $5 / 9(55.6 \%)$ lesions $(\mathrm{P}<0.05)$, and the proportion of score 3 increased to $3 / 4$ (75.0) in SCC samples $(\mathrm{P}<0.05)$.

PD-L1 expression in normal epithelium, CIN, and SCC samples are shown in Supplementary Table S4. All of the normal squamous epithelium, CIN1, and CIN2 samples were negative. Limited samples of CIN3 $(1 / 9 ; 11.1 \%)$ and SCC $(2 / 4 ; 50 \%)$ showed positive staining for PD-L1.

\section{No association between the expression level of HLA class I mRNA and CIN degree or immunohistochemical HLA class I pattern}

The HLA class I mRNA expression level normalized to $\beta$-actin was rather stable in CIN1, CIN2, and CIN3 without statistical significance (Fig. 2a). Although the HLA class I mRNA expression level tended to be lower in the $\mathrm{N}$ pattern of immunohistochemical findings for HLA class I than those in other types (M1, M2, and H pattern), there was no statistical significance (Fig. 2b).

\section{Alteration of the CIN grade and the HLA class I expression pattern during the follow-up period}

Fourteen patients, who were examined twice during the follow-up period, were further analyzed. The CIN grade was altered in 5 cases, and the IHC expression pattern of HLA class I was altered in 8 cases. The correlation between 
a

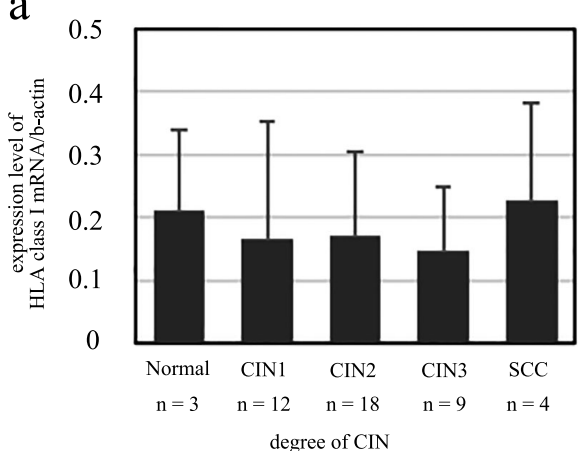

b

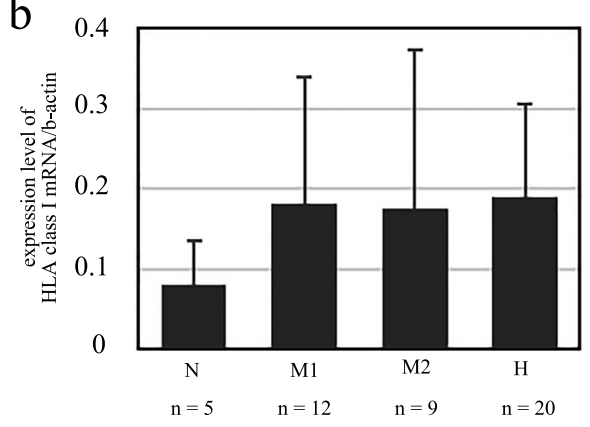

IHC pattern of HLA class I

Fig. 2. Expression level of HLA class I mRNA normalized to $\beta$-actin. Expression levels of HLA class I mRNA were compared to (a) the CIN grade, and (b) the immunohistochemical pattern of HLA class I.

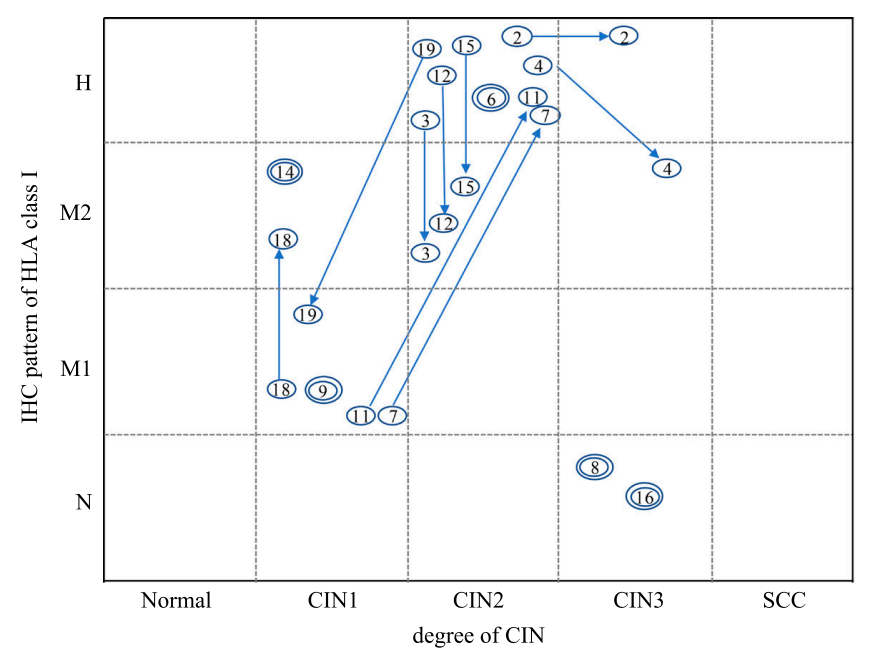

Fig. 3. Alteration of the $\mathrm{CIN}$ grade and immunohistochemical pattern of HLA class I expression. The case numbers in the circles are plotted for the first and second examinations. The arrow shows alterations from the first to the second examination. Double circles indicate that there is no change between the first and second examinations.

IHC expression pattern of HLA class I and the CIN grade between the first and second examinations is shown in Fig. 3. All of CIN2 showed $\mathrm{H}$ pattern at first examination, and 4/7 cases and 1/7 cases altered to M2 pattern and M1 pattern, respectively. Immunohistochemical findings of a representative case of CIN2 with altered HLA class I from $\mathrm{H}$ pattern to M2 pattern is shown in Fig. 4.

The rate of change of HLA class I mRNA expression level was calculated and compared in each case (Table 2). Change of CIN degree between first and second examination from CIN1 to CIN2 to CIN3 was defined as progressive, and reverse change was defined as regressive. The progressive group (PG) or no-change group (NCG) of CIN grade showed higher rates of change of HLA class I mRNA expression level than those of the regressive group (RG) of CIN grade, though there was no statistical significance (Fig. 5a).

According to the results of the correlation analysis between the IHC pattern and the CIN grade, alteration of
IHC pattern from $\mathrm{N}$ to $\mathrm{M} 1$ to $\mathrm{M} 2$ to $\mathrm{H}$ pattern was defined as progressive (Supplementary Fig. S1). The IHC progressive groups (IHC-PG) in the second examination showed a significantly higher rate of change than those of the IHC regressive groups (IHC-RG) $(\mathrm{P}=0.0369)$ (Fig. 5b).

\section{Discussion}

The present study demonstrated that the immunohistochemical pattern of HLA class I glycoproteins was different in each grade of CIN lesions. Epithelial cells expressing HLA class I were limited to the basal part of the epithelium (M1 pattern) in CIN1, and this pattern decreased in CIN2. In CIN3, the expression of HLA class I became negative. A heterogeneous positive pattern ( $\mathrm{H}$ pattern) was rarely detected in CIN1 but was increased in CIN2 and CIN3, and all SCC samples showed the H pattern. During the clinical follow-up period, the IHC pattern was altered more frequently than the CIN grade. Especially, $\mathrm{H}$ pattern detected in CIN2 were high alterative to M2 pattern. There was no report that analyzed IHC patterns in dysplastic lesion of lower grade (CIN1) to CIN3, nor report that demonstrated the changes of IHC pattern were alterable during clinical course, to our knowledge.

A decrease in HLA class I antigen expression has been reported in cervical SCC and carcinoma in situ by immunohistochemistry or flow cytometry and by analysis of loss of heterozygosity (LOH). Vermeulen et al. reported that $91 \%$ of CIN3 or SCC showed decreased (negative or weak) expression of HLA class I by IHC [18]. The proportion of LOH at the chromosome 6p21.3 (HLA locus) increased from $82 \%$ [18] to $96 \%[11,12]$. Altered phenotypes of HLA class I were categorized into type I (total loss of HLA), type II (haplotype loss), type III (locus loss), and type IV (allelic loss). The proportion of each type was $10 \%, 50 \%, 10 \%$, and $17 \%$, respectively $[6,7]$. Such frequent alterations of HLA class I phenotypes may affect the decreased immunohistochemical expression findings. The present study showed that the $\mathrm{N}$ pattern increased in CIN3, which supported the higher incidence of LOH in CIN3 and SCC reported previously. The possible explanations 

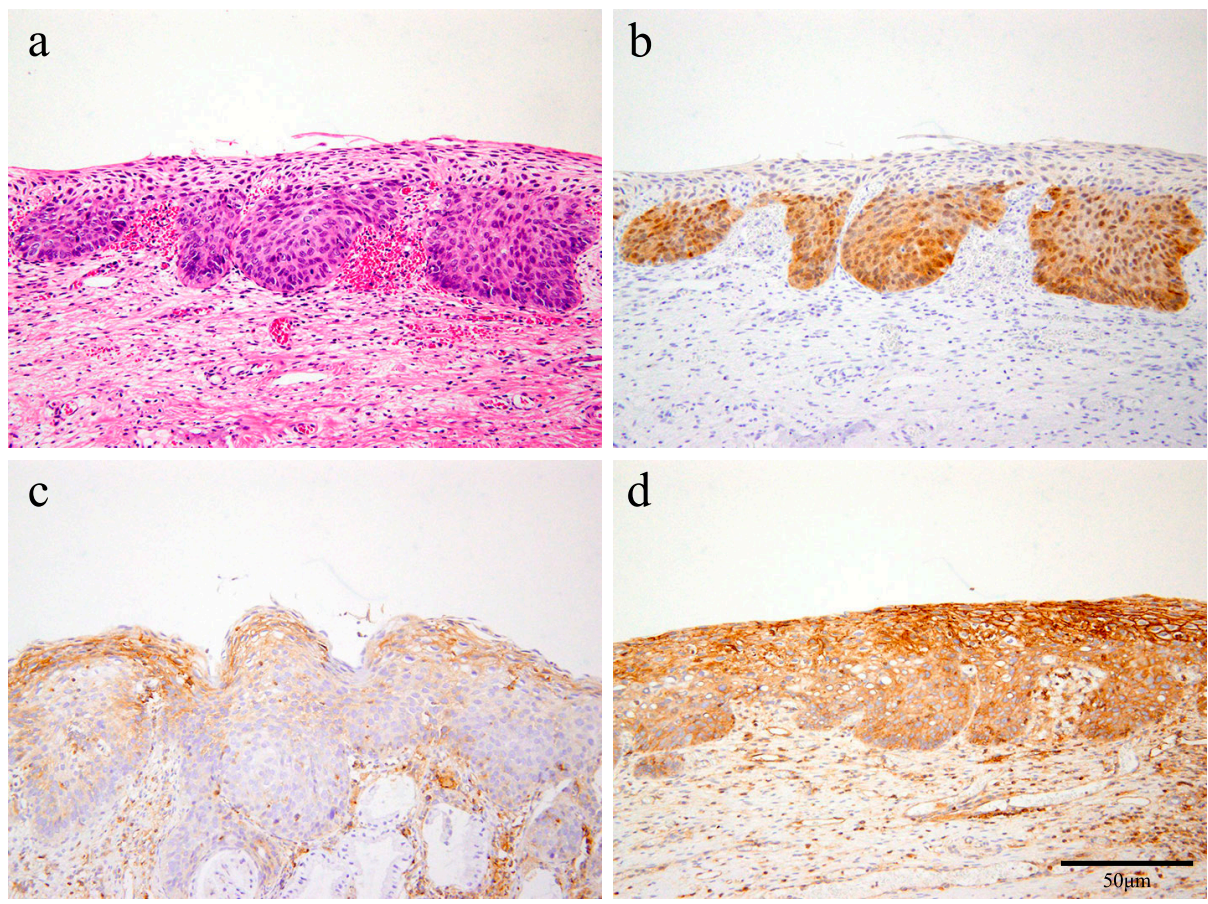

Fig. 4. Immunohistochemical findings of a representative case of CIN2. Pathological features of a representative case of CIN2 (HE stain) (a), immunohistochemical findings of p16 (b) and HLA class I of $\mathrm{H}$ pattern at first examination (c) and M2 pattern at second examination (d) are shown. Bar $=50 \mu \mathrm{m}$.

Table 2. Alterations of the CIN, IHC pattern, and mRNA expression level of HLA class I

\begin{tabular}{|c|c|c|c|c|c|c|c|}
\hline \multirow{2}{*}{ Case number } & \multicolumn{3}{|c|}{ Degree of CIN } & \multicolumn{3}{|c|}{ IHC pattern of HLA class I } & \multirow{2}{*}{$\begin{array}{l}\text { Changed rate of mRNA expression level } \\
\text { of HLA class I } 2^{(-\Delta \mathrm{Ct} 2 \mathrm{nd})} / 2^{(-\Delta \mathrm{Ct} 1 \mathrm{st})}\end{array}$} \\
\hline & 1 st & 2 nd & Group & $1 \mathrm{st}$ & 2 nd & Group (IHC) & \\
\hline 9 & 1 & 1 & NCG & M1 & M1 & NCG & 2.11 \\
\hline 18 & 1 & 1 & NCG & M1 & M2 & PG & 4300 \\
\hline 14 & 1 & 1 & NCG & M2 & M2 & NCG & 3.12 \\
\hline 7 & 1 & 2 & PG & M1 & $\mathrm{H}$ & PG & 1.17 \\
\hline 11 & 1 & 2 & PG & M1 & $\mathrm{H}$ & PG & 3304 \\
\hline 19 & 2 & 1 & RG & $\mathrm{H}$ & M1 & RG & 0.47 \\
\hline 6 & 2 & 2 & NCG & $\mathrm{H}$ & $\mathrm{H}$ & NCG & 0 \\
\hline 3 & 2 & 2 & NCG & $\mathrm{H}$ & M2 & RG & 0.36 \\
\hline 12 & 2 & 2 & NCG & $\mathrm{H}$ & M2 & RG & 0.01 \\
\hline 15 & 2 & 2 & NCG & $\mathrm{H}$ & M2 & $\mathrm{RG}$ & 0.49 \\
\hline 2 & 2 & 3 & PG & $\mathrm{H}$ & $\mathrm{H}$ & NCG & 19756 \\
\hline 4 & 2 & 3 & PG & $\mathrm{H}$ & M2 & RG & 0.71 \\
\hline 8 & 3 & 3 & NCG & $\mathrm{N}$ & $\mathrm{N}$ & NCG & 1.27 \\
\hline 16 & 3 & 3 & NCG & $\mathrm{N}$ & $\mathrm{N}$ & NCG & 0.01 \\
\hline
\end{tabular}

CIN, cervical intraepithelial neoplasia; IHC, immunohistochemistry. PG, progressive group; NCG, no changed group; RG, regressive group.

for the heterogeneous $(\mathrm{H})$ pattern are as follows: (1) HLA Class I alterations including haplotype loss, locus loss, and allelic loss, which could produce incomplete proteins consisting of HLA Class I, and/or (2) up or down regulation of HLA class I gene occurring in each cell. The present study showed that the incidence of the $\mathrm{H}$ pattern tended to increase since CIN2, and was detected frequently in SCC with a statistical significance.
The present study showed that CIN1 or CIN2 could be up or down graded in 5/14 (35.7\%) cases, and that the IHC pattern was altered more frequently during the clinical process in $8 / 14(57.1 \%)$ cases. The most frequent pattern change was from $\mathrm{H}$ to $\mathrm{M} 2$ or $\mathrm{M} 1$ pattern $(5 / 7$ cases of CIN2), and the next frequent pattern change was from the M1 or M2 to H pattern ( $2 / 5$ cases of CIN1). These results suggest that the $\mathrm{H}$ pattern observed in CIN2 is a highly 
a

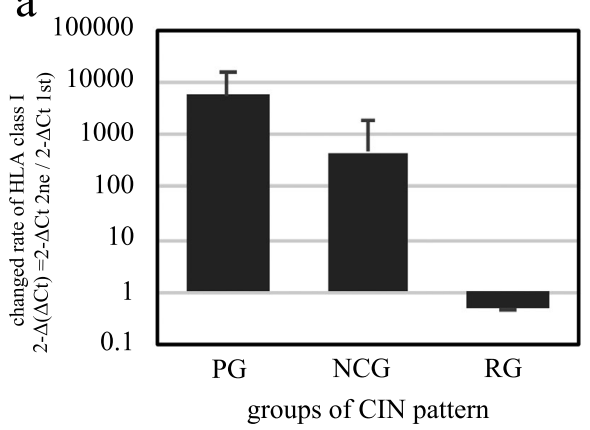

b

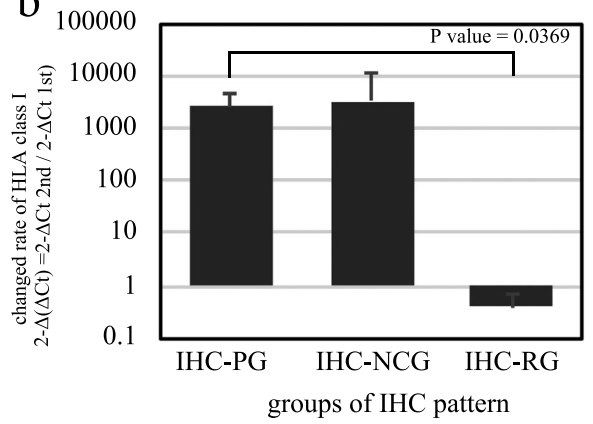

Fig. 5. The changed rate of HLA class I mRNA expression. The rate of change of HLA class I mRNA expression between the first and second examinations were compared among the progressive group (PG), no-change group (NCG), and regressive group (RG) for (a) the CIN grade, and (b) the immunohistochemical expression pattern for HLA class I.

alterable phenotype, and it was unlikely due to constant genetic alterations. Because, if $\mathrm{H}$ pattern detected at the first examination was a result of genetic loss (haplotype, locus and allelic loss), it was hardly to occur that CIN lesions at second examination recovered genetic loss and produced protein products of HLA class I expressed. The present study revealed that CIN2 is an unstable phase based on this heterochronous observation, and it was unlikely that $\mathrm{H}$ pattern detected in CIN2 was results of the permanent genetic loss of HLA class I.

IHC results showed that CIN2 consisted of various phenotypes. Holowaty et al. reported that the regression rates of both $\mathrm{CIN} 1$ and $\mathrm{CIN} 2$ were higher than the progression rates, and CIN1 regressed more frequently than CIN2 naturally [10]. CIN2 is clinically problematic because the treatment of cases with CIN2 differs between medical institutions [5]. Furthermore, LEEP and conization for CIN2 increase the risk of preterm labor [15]. However, the predictive markers of the progression and regression of CIN2 are still unknown. The present study suggests that the alteration in the HLA class I pattern was more frequent than that in the CIN grade, and is associated with the rate of change of HLA class I mRNA expression level. Therefore, it may be possible to distinguish progressive CIN2 cases from regressive ones by examining the HLA class I pattern and the rate of change of HLA class I mRNA expression level. Consequently, the potential risk of progression to invasive cervical cancer may decrease and overtreatment for CIN can be avoided.

The present study showed no relationship between PD-L1 and CIN degree. Previous studies have shown that upregulation of HLA class II and several types of polymorphisms have possible roles in the carcinogenesis of cervical SCC; however, the correlation has not been demonstrated in the pre-malignant phase. The PD-L1 and PD1 complex are one of the mechanisms of the acquired immune system. Therefore, it is not surprising that there is no expression of PD-L1 in the pre-malignant phase of CIN, when there is no tumor immunity established based on the acquired immune system.

In conclusion, the present study showed that the IHC pattern of HLA class I expression is associated with the CIN grade, and is alterable during the clinical course, especially in CIN2.

\section{Conflicts of interest}

The authors have no conflicts of interest that may be related to the content of the manuscript.

\section{Acknowledgments}

The authors would like to thank Mrs. Yukari Hirotani, Division of Oncologic Pathology, Department of Pathology and Microbiology, for technical support for immunohistochemistry, and to Prof. Kei Kawana, Department of Obstetrics and Gynecology, for providing medical suggestions regarding uterine cervical cancer.

\section{References}

1. Center for Cancer Control and Information Services, N. C. C. (2020) CANCER STATISTICS IN JAPAN-2019.

2. Cheng, L., Guo, Y., Zhan, S. and Xia, P. (2018) Association between HLA-DP Gene Polymorphisms and Cervical Cancer Risk: A Meta-Analysis. Biomed. Res. Int. 2018; 7301595.

3. Cromme, F. V., Meijer, C. J., Snijders, P. J., Uyterlinde, A., Kenemans, P., Helmerhorst, T., et al. (1993) Analysis of MHC class I and II expression in relation to presence of HPV genotypes in premalignant and malignant cervical lesions. $\mathrm{Br}$. J. Cancer 67; 1372-1380.

4. Engelmark, M., Beskow, A., Magnusson, J., Erlich, H. and Gyllensten, U. (2004) Affected sib-pair analysis of the contribution of HLA class I and class II loci to development of cervical cancer. Hum. Mol. Genet. 13; 1951-1958.

5. Fuchs, K., Weitzen, S., Wu, L., Phipps, M. G. and Boardman, L. A. (2007) Management of cervical intraepithelial neoplasia 2 in adolescent and young women. J. Pediatr. Adolesc. Gynecol. 20; 269-274.

6. Garrido, F., Ruiz-Cabello, F., Cabrera, T., Perez-Villar, J. J., 
Lopez-Botet, M., Duggan-Keen, M., et al. (1997) Implications for immunosurveillance of altered HLA class I phenotypes in human tumours. Immunol. Today 18; 89-95.

7. Garrido, F. and Algarra, I. (2001) MHC antigens and tumor escape from immune surveillance. Adv. Cancer Res. 83; $117-158$.

8. Georgopoulos, N. T., Proffitt, J. L. and Blair, G. E. (2000) Transcriptional regulation of the major histocompatibility complex (MHC) class I heavy chain, TAP1 and LMP2 genes by the human papillomavirus (HPV) type 6b, 16 and 18 E7 oncoproteins. Oncogene 19; 4930-4935.

9. Glew, S. S., Duggan-Keen, M., Cabrera, T. and Stern, P. L. (1992) HLA class II antigen expression in human papillomavirus-associated cervical cancer. Cancer Res. 52; 4009-4016.

10. Holowaty, P., Miller, A. B., Rohan, T. and To, T. (1999) Natural history of dysplasia of the uterine cervix. J. Natl. Cancer Inst. 91; 252-258.

11. Koopman, L. A., Mulder, A., Corver, W. E., Anholts, J. D., Giphart, M. J., Claas, F. H., et al. (1998) HLA class I phenotype and genotype alterations in cervical carcinomas and derivative cell lines. Tissue Antigens. 51; 623-636.

12. Koopman, L. A., Corver, W. E., van der Slik, A. R., Giphart, M. J. and Fleuren, G. J. (2000) Multiple genetic alterations cause frequent and heterogeneous human histocompatibility leukocyte antigen class I loss in cervical cancer. J. Exp. Med. 191; 961-976.

13. Koromilas, A. E., Li, S. and Matlashewski, G. (2001) Control of interferon signaling in human papillomavirus infection. Cytokine Growth Factor Rev. 12; 157-170.

14. Kurman, R. J., Carcangiu, M. L., Herrington, C. S. and Young, R. H. (2014) WHO Classification of Tumours of Female Reproductive Organs, 4th ed., International Agency for Research on Cancer, Lyon.

15. Kyrgiou, M., Koliopoulos, G., Martin-Hirsch, P., Arbyn, M., Prendiville, W. and Paraskevaidis, E. (2006) Obstetric outcomes after conservative treatment for intraepithelial or early invasive cervical lesions: systematic review and meta-analysis. Lancet 367; 489-498.

16. Li, W., Deng, X. M., Wang, C. X., Zhang, X., Zheng, G. X., Zhang, J., et al. (2010) Down-regulation of HLA class I antigen in human papillomavirus type $16 \mathrm{E} 7$ expressing $\mathrm{HaCaT}$ cells: correlate with TAP-1 expression. Int. J. Gynecol. Cancer 20; 227-232.

17. Mikyskova, R., Bubenik, J., Vonka, V., Smahel, M., Indrova, M., Bieblova, J., et al. (2005) Immune escape phenotype of HPV16-associated tumours: MHC class I expression changes during progression and therapy. Int. J. Oncol. 26; 521-527.

18. Vermeulen, C. F., Jordanova, E. S., Zomerdijk-Nooijen, Y. A., ter Haar, N. T., Peters, A. A. and Fleuren, G. J. (2005) Frequent HLA class I loss is an early event in cervical carcinogenesis. Hum. Immunol. 66; 1167-1173.

19. Wei, L. Z., Wang, H. L., Liu, X., Lu, Y. P., Xu, F., Yuan, J. Q., et al. (2014) Meta-analysis on the relationship between HLA-DRB1 gene polymorphism and cervical cancer in Chinese population. PLoS One 9; e88439.

20. Yoshino, T., Muro, K., Yamaguchi, K., Nishina, T., Denda, T., Kudo, T., et al. (2015) Clinical Validation of a Multiplex Kit for RAS Mutations in Colorectal Cancer: Results of the RASKET (RAS KEy Testing) Prospective, Multicenter Study. EBioMedicine 2; 317-323.

This is an open access article distributed under the Creative Commons License (CC-BY-NC), which permits use, distribution and reproduction of the articles in any medium provided that the original work is properly cited and is not used for commercial purposes. 